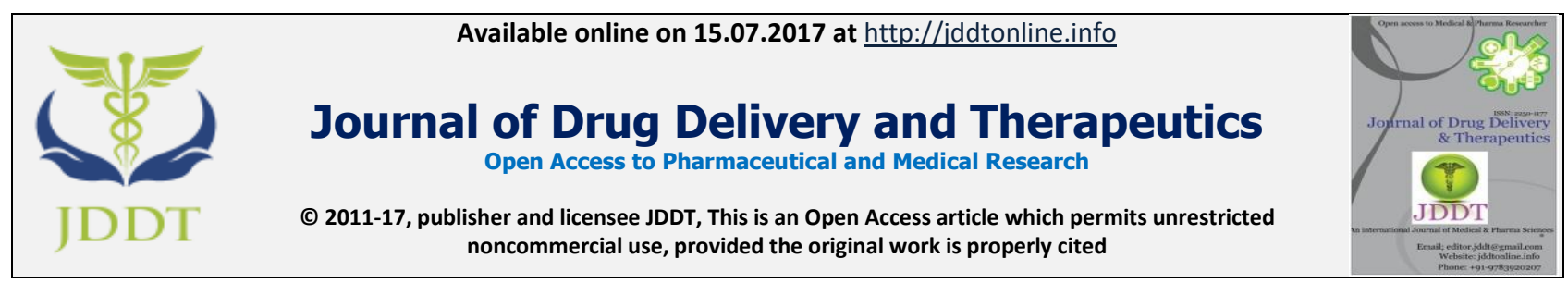

Open 6 Access

Review Article

\title{
LIQUISOLID COMPACTS: A PROMISING APPROACH FOR SOLUBILITY ENHANCEMENT
}

\author{
Pawar J.D. ${ }^{1 *}$, Jagtap R.S. ${ }^{2}$, Doijad R.C ${ }^{1}$, Pol S .V ${ }^{1}$, Desai J. R. ${ }^{1}$, Jadhav V.V. ${ }^{1}$, Jagtap S.R. ${ }^{2}$ \\ ${ }^{1}$ Department of Pharmaceutics, Shree Santkrupa College Of pharmacy, Ghogaon, Karad, Maharashtra, India \\ ${ }^{2}$ Departments of Pharmaceutics, Annasaheb Dange College of Pharmacy, Ashta, Dist. Sangli, Maharashtra-416 301, India
}

\begin{abstract}
At present $40 \%$ of the drugs within the development pipelines, and approximately $60 \%$ of the drugs coming directly from synthesis area unit poorly soluble. Solubility is one of the important parameter to obtain desired concentration of drug in systemic circulation. Liquisolid compacts technique is a new and promising approach to overcome this consequence and that can change the dissolution rate of water insoluble drugs and increase the bioavailability of the drugs. This technique is an efficient method for formulating water insoluble and water soluble drugs. This technique relies upon the admixture of drug loaded solutions with applicable carrier and coating materials. Liquisolid system is characterized by flow behaviour, wettability, powder bed hydrophilicity, saturation solubility, drug content, differential scanning calorimetry, Fourier transform infra-red spectroscopy, powder X-ray diffraction, scanning electron microscopy, in-vitro release and in-vivo evaluation. This review article explains the preparation, classification and application of liquisolid system.
\end{abstract}

Key word: Liquisolid system, water insoluble drug, carrier material, coating material.

Article Info: Received 17 May, 2017; Review Completed 28 June, 2017; Accepted 08 July, 2017; Available online 15 July, 2017

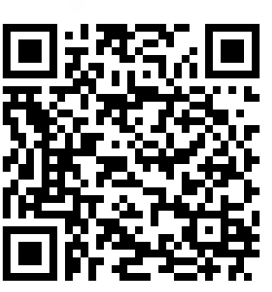

Cite this article as:

Pawar J.D., Jagtap R.S., Doijad R.C., Pol S.V., Desai J. R., Jadhav V.V., Jagtap S.R., Liquisolid compacts: A promising approach for solubility enhancement, Journal of Drug Delivery and Therapeutics. 2017; 7(4):6-11

DOI: http://dx.doi.org/10.22270/jddt.v7i4.1466

*Address for Correspondence

Janardhan D. Pawar, Department of Pharmaceutics, Shree Santkrupa College Of pharmacy, Ghogaon, Karad, Maharashtra, India. E-mail: *janardhanpawar3@gmail.com

\section{INTRODUCTION}

The oral route is the most preferred route of drug administration due to the ease, high patient acceptance, and low cost production. The drug must be presented in solution form for absorption through gastrointestinal tract (GIT) when given orally.The solubility and dissolution behaviour of a drug is the key determinants of its oral Bioavailability ${ }^{1}$. Release improvement of poorly soluble drugs is rise achieved by a rise of the drug surface area, the drug solubility, or by formulating the drug in its dissolved state. Numerous techniques are used to formulate oral drug delivery system that might enhance the dissolution profile and successively, the absorption potency of water insoluble drugs likes micronization, adsorption onto high surface area transporters, lyophilization, co-grinding, formulation of inclusion complexes, solubilization by surfactants, solid dispersions, solid solutions, hydrotrophy, inclusion of the drug resolution or liquid drug into soft gelatin capsules, and cosolvency and liquisolid compact technology ${ }^{2}$. The Liquisolid compact concept described by Spireas et. al. in this system oily liquid drug and solution or suspension of water insoluble drug in to non-volatile solvent ${ }^{3}$. Liquisolid technology, a liquid is also reworked into a free flowing, without delay compressible and apparently dry powder by easy physical mixing with elect excipients named the carrier and coating material. The liquid portion, which can be a liquid drug, a drug suspension or a drug solution in to suitable non-volatile liquid vehicles, is combined into the carrier material. The foremost promising and new technique for supporting dissolution is that the formation of liquisolid tablets among the numerous novel 
techniques. Liquisolid compacts promotes dissolution rate of water insoluble drugs to a greater extent and additionally enhances the drug flow property ${ }^{4,5}$.

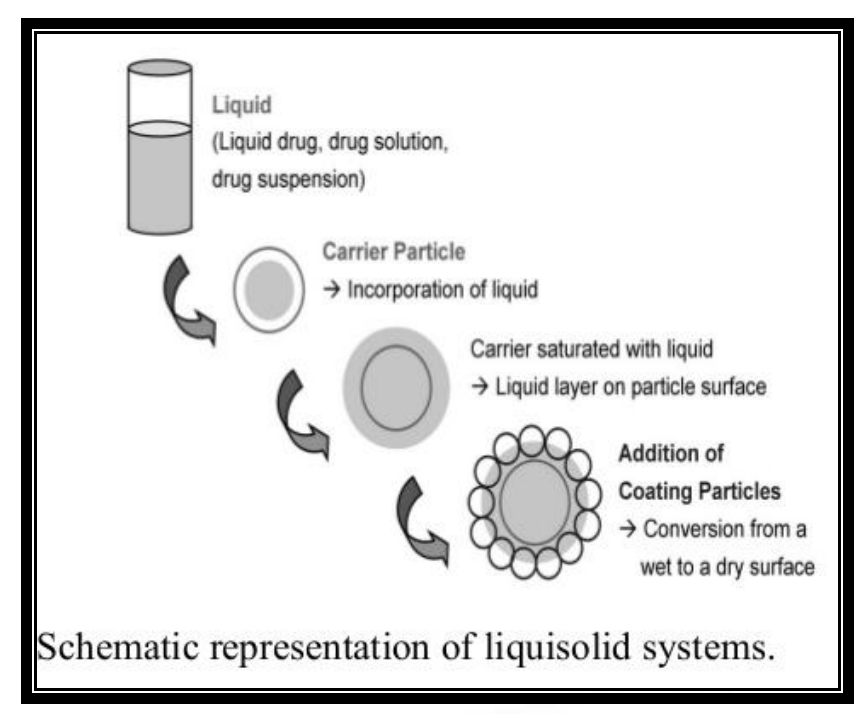

Fig. 1: Schematic representation of liquisolid system.

\section{CLASSIFICATION}

A. Based on the liquid medication, it divided into three sub groups:

\section{Powdered drug solutions}

\section{Powdered drug suspensions}

\section{Powdered liquid drug}

The major two is also made from the conversion of drug solutions or drug suspensions (e.g. gemfibrozil suspension in Polysorbate 80), and therefore the formulation of liquid drugs (e.g. clofibrate, valproic acid, liquid vitamins, etc.), into liquisolid systems.

B. Based on the formulation technique, liquisolid systems is classified into two groups,

1. Liquisolid compacts: It refers to immediate sustained release tablets or capsules that are described under liquisolid systems.

2. Liquisolid microsystems: It refers to capsules ready by liquisolid systems with the inclusion of an additive resultant in a unit size that may be as much as five times less than that of a liquisolid compact ${ }^{6}$.

\section{Theory of Liquid Solid Systems:}

A powder can retain only restricted amounts of liquid whereas maintaining acceptable flow and compression properties. To calculate the essential amounts of powder excipients (carrier and coating materials) a mathematical approach for the formulation of liquisolid systems has been developed by Spireas. This approach is predicated on the flowable ( $\Phi$ value) and compressible ( $\Psi$-number) liquid retention potential introducing constants for each powder/liquid combination. The $\Phi$-value of a powder represents the determined amount of a given non-volatile liquid which will be maintained within its bulk [w/w] whereas maintaining asuitable flowability. The flowability may be determined from the powder flow or by measurement of the angle of repose ${ }^{7}$.

The $\Psi$-number of a powder is defined as the maximum amount of liquid the powder can retain inside its bulk [w/w] while maintaining acceptable compactability resulting in compacts of sufficient hardness with no liquid permeable out throughout compression.

The compactability determined by the pactisity, which describes the maximum (plateau) crushing strength of a one-gram tablet compacted at sufficiently high compression forces.

The terms "acceptable flow and compression properties" imply the specified so preselected flow and compaction properties that has to to be met by the ultimate word liquisolid formulation.

Depending on the excipient ratio $(\mathrm{R})$ of the powder substrate an reasonably flowing and compressible liquisolid system can be obtained only if a maximum liquid load on the carrier material is not exceeded. This liquid/carrier ratio is termed "liquid load factor $\mathrm{Lf}^{\text {" }}$ [w/w] and is defined as the weight ratio of the liquid formulation (W) and the carrier material (Q) in the system:

$$
\mathrm{Lf}=\mathrm{W} / \mathrm{Q} \quad \text { Eq. (1) }
$$

$\mathrm{R}$ represents the quantitative relation between the weights of the carrier (Q) and also the coating (q) material gift within the formulation:

$$
\mathrm{R}=\mathrm{Q} / \mathrm{q} \quad \text { Eq. (2) }
$$

The liquid load factor that ensures acceptable flowability ( $\Phi \mathrm{Lf}$ ) can be determined by:

$$
\Phi L f=\Phi+\varphi \cdot(1 / R) \quad \text { Eq. (3) }
$$

where $\Phi$ and $\varphi$ are the $\Phi$-values of the carrier and coating material, respectively. Similarly, the liquid ratio for production of liquisolid systems with acceptable compactability ( $\Psi \mathrm{Lf})$ is decided by:

$$
\Psi \mathrm{Lf}=\Psi+\psi \cdot(1 / \mathrm{R}) \quad \text { Eq. (4) }
$$

where $\Psi$ and $\psi$ are the $\Psi$-numbers of the carrier and coating material, respectively.In Table-1 sample of liquisolid formulation parameters of different powder excipients with usuallyused liquid vehicles are listed.

Therefore, the optimum liquid load factor (Lo) needed to get tolerably flowing and compressible liquisolid systems are adequate to either $\Phi$ Lfor $\Psi L f$, whichever represents the lower value.

As soon as the optimum liquid load factor is determined, the appropriatequantities of carrier (Qo) and coating (qo) material required to convert a given amount of liquid formulation (W) into an acceptably flowing and compressible liquisolid system is additionally calculated as follows:

$$
\begin{gathered}
\mathrm{Q} 0=\mathrm{W} / \mathrm{Lo}-----(5) \\
\text { And } \mathrm{q} 0=\mathrm{Q} 0 / \mathrm{R}-------(6)
\end{gathered}
$$


Table 1: Liquisolid formulation parameters of various powder excipients with commonly used liquid vehicles

\begin{tabular}{|c|c|c|c|c|}
\hline \multirow{2}{*}{ Powder Excipient or System } & \multicolumn{2}{|c|}{ Ф-values } & \multicolumn{2}{c|}{-numbers } \\
\cline { 2 - 5 } & Propylene glycol & PEG 400 & Propylene glycol & PEG 400 \\
\hline Avicel pH 102 & 0.16 & 0.005 & 0.224 & 0.242 \\
\hline Avicel pH 200 & 0.26 & 0.02 & 0.209 & 0.232 \\
\hline Cab-O-Sil M5 M5(silica)* With Avicel pH 102 & 3.31 & 3.26 & 0.560 & 0.653 \\
\hline Cab OSilM5(silica)* With Avicel pH 200 & 2.57 & 2.44 & 0.712 & 0.717 \\
\hline
\end{tabular}

The validity and applicability of the above mentioned principles have been tested and verified by producing liquisolid compacts possessing acceptable flow and compaction properties ${ }^{8,9}$.

\section{MECHANISMS OF ENHANCED DRUG RELEASE FROM LIQUISOLID SYSTEMS}

Several mechanisms of increased drug release have been proposed for liquisolid systems. The three main mechanisms include increased surface area of drug, increased aqueous solubility of the drug, and improved wettability of the drug particles. Formation of a complex between the drug and excipients or any changes in crystalline of the drug could be ruled out using DSC and XRPD measurements.
a) Increased Aqueous Solubility
b) Increased Drug Surface Area
c) Increased Wettability

\section{a) Increased Drug Surface Area}

If the drug within the liquisolid system is totally dissolved within the liquid vehicle it is situated in the powder substrate still in a solubilized, molecularly spread state. Therefore, the surface area of drug available for release is way larger than that of drug particles inside directly compressed tablets. Accordingly, with increasing drug content surpassing thesolubility limit and so, increasing fraction of undissolved drug within the liquid vehicle the discharge rate decreases ${ }^{10}$.

\section{b) Increased Aqueous Solubility of the Drug}

In addition to the primary mechanism of drug release improvement it is expected that cs, the solubility of the drug might be increase with liquisolid system. In fact the relatively small amount of liquid vehicle in a liquisolid compact is not adequate to extend the general solubility of the drug within the binary compound dissolution medium. However at the solid liquid interface between an distinct liquisolid primary particles and the release medium it is promising that in this micro environment the quantity of liquid vehicle spreading out of a single liquisolid particle together with the drug molecules might be adequate to extend the aqueous solubility of the drug if the liquid vehicle acts as co solvent ${ }^{11}$.

\section{c) Increased Wettability}

Due to the very fact that the liquid vehicle will either act as surface chemical agent or contains a low surface tension, wetting of the liquisolid primary particles is improved. Wettability of these systems has been confirmed by measurement of contact angles and water rising times ${ }^{12}$.

\section{GENERAL PROCEDURE FOR PREPARATION OF LIQUISOLID COMPACT TABLETS}

1. A drug substance should be initially dissolved or suspended in a suitable non-volatile solvent system to produce a drug solution or drug suspension of desired concentration.

2. Then a mixture of carrier or different polymers and excipients were added to the above liquid medication under continuous mixing in a mortar. These amounts of the carrier and excipients areenough to maintain acceptable flow and compression properties.

3. To the above binary mixture disintegrant like sodium starch glycolate and other reaming additives were added according to their application and mixed for a period of 10 to $20 \mathrm{~min}$. in a mortar.

4. The final mixture was compressed using the manual tableting machine to achieve tablet hardness.

5. Characterize the final liquisolid granules for solubility, dissolution, flowability, compressibility and other physicochemical properties ${ }^{13}$.

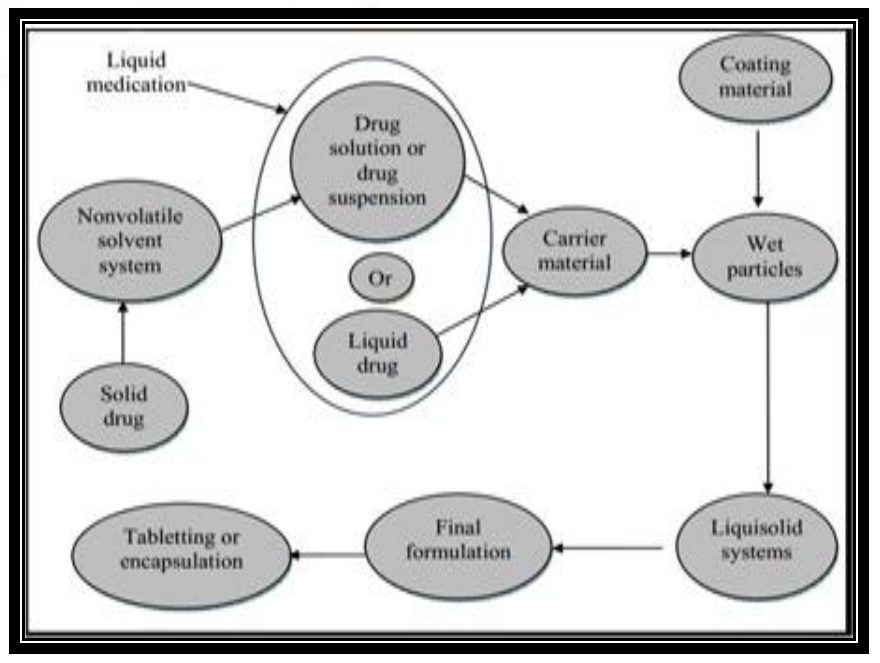

Figure 2: Schematic Diagram Representing Preparation of Liquisolid Compacts 


\section{COMPONENTS OF LIQUISOLID COMPACT FORMULATIONS}

\section{Non-volatile Solvent}

Non-volatile Solvent should be Inert, high boiling point, ideally water-miscible and not extremely viscous organic solvent systems and compatible with having ability to solubilise the drug. The non-volatile solvent acts as a binding agent within the liquisolid formulation. Various non-volatile solvents used in the formulation of liquisolid compact. Eg. Polyethylene glycol 200 and 400 , glycerin, polysorbate 80 and propylene glycol.

\section{Disintegrant}

Super disintigrants rises the rate of drug release, water solubility and wettability of liquisolid granules. Mostly super disintigrants like sodium starch glycolate and cross povidone.

\section{Carrier Materials}

Carrier material should be porous material possessing adequate absorption properties which contributes in liquid absorption. The carrier and coating materials will retain only solely bound amounts of liquid and at the constant time maintain acceptable flow and compression properties therefore, increasing moisture content of carrier leads to decreased powder flow ability These contain grades of microcrystalline cellulose such as avicel PH 102 and avicel PH 200.

\section{Coating Materials}

Coating material should be a material possessing fine and extremely surface-assimilative particles that contributes in covering the wet carrier particles and displaying a dry looking powder by adsorbing any excess liquid. Coating material is required to cover the surface and maintain the powder flowability34.Coating material includes silica (Cab-O-Sil) M520, 35, Aerosil 20030, Syloid, 244FP 20,35 etc ${ }^{14,15}$.

Examples of some drugs that can be incorporated into liquisolid systems:

\begin{tabular}{|c|c|}
\hline$*$ & Chlorpheniramine \\
\hline$*$ & Digoxin \\
\hline$*$ & Nifedipine \\
\hline$*$ & Clofibrate \\
\hline 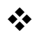 & Gemfibrozil \\
\hline 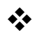 & Etoposide \\
\hline$*$ & Carbamazepine \\
\hline$*$ & Hydrochlorothiazide \\
\hline 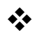 & Methyclothiazide \\
\hline 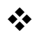 & Spironolactone \\
\hline$*$ & Hydrocortisone \\
\hline$*$ & Piroxicam \\
\hline 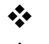 & Indomethacin \\
\hline$*$ & Ibuprofen \\
\hline
\end{tabular}

\section{PRE-COMPRESSION STUDIES OF THE LIQUISOLID SYSTEM}

\section{Flow Properties of the Liquisolid System:}

The flow properties of the liquisolid systems were calculable by determining the angle of repose, Carr's index, and Hausner's ratio.

\section{Angle of repose:}

The angle of repose physical mixtures of liquisolid compacts were determined by fixed funnel method. The accurately weighed physical mixtures of liquisolid compacts were taken in a funnel. The height of the funnel was adjusted in such how that the tip of the funnel simply touches the apex of the heap of the powder. The powder was allowed to flow through the funnel freely into the surface. The height and diameter of the powder cone was measured and angle of repose was calculated.

$\operatorname{Tan} \theta=\mathrm{h} / \mathrm{r}$

Where, $\theta$ is the angle of repose, $h$ is the height, $r$ is the radius.

Values for angle of repose $\leq 300$ usually indicate a free flowing material and angles $\geq 400$ suggest a poorly flowing material. 25- 30 showing excellent flow properties,31-35 showing good flow properties, 36-40 showing fair flow properties, 41-45 showing passable flow properties.

\section{Bulk Density:}

The loose bulk density and tapped density were determined by using bulk density apparatus. Apparent bulk density was determined by pouring the blend into a graduated cylinder. The bulk volume $(\mathrm{Vb})$ and weight of the powder (M) was firm. The bulk density was calculated using the formula:

\section{$\mathrm{Db}=\mathrm{M} / \mathrm{Vb}$}

Where, $\mathrm{M}$ is the mass of powder

$\mathrm{Vb}$ is bulk volume of powder

\section{Tapped Density:}

The measuring cylinder containing a known mass of blend was tapped for a fixed time. The minimum volume $(\mathrm{Vt})$ occupied within the cylinder and therefore the weight (M) of the mix was measured. The tapped density was calculated using the Formula:

$\mathrm{Dt}=\mathrm{M} / \mathrm{Vt}$

Where, $\mathrm{M}$ is the mass of powder

$\mathrm{Vt}$ is tapped volume of powder

\section{Carr's Index (\%):}

The compressibility index has been proposed as an indirect measure of bulk density, size and shape, surface area, moisture content and cohesiveness of material because all of these ca influence the observed compressibility index. The simplest method for measurement of free flow of powder is Carr's Index, a indication of the benefit with which a material can be induced to flow is given by Carr's index (CI) which is calculated as follows:

CI $(\%)=[($ Tapped density - Bulk density $) /$ Tapped density] x 100

The value below $15 \%$ indicates a powder with sometimes offers rise to good flow characteristics, where as above $25 \%$ indicates poor flow ability. 


\section{Hausner's Ratio:}

Hausner's ratio is an indirect index of ease of powder flow. It is calculated by the following formula.

Hausner's Ratio=Tapped density $(\rho t) /$ Bulk density $(\rho b)$

Where $\rho t$ is tapped density and $\rho b$ is bulk density. Lower Hausner's ratio $(<1.25)$ indicates better flow properties than higher ones, between 1.25 to 1.5 showing moderate flow properties and more than 1.5 poor flow ${ }^{16,17}$.

\section{POST COMPRESSION STUDIES OF LIQUI- SOLID COMPACTS}

\section{Hardness:}

Monsanto hardness tester can be used for the determination of the hardness. The tablet to be tested was held between a fixed and moving jaw and reading of the indicator adjusted to zero. The force applied to the edge of the tablet is gradually increased by moving the screw knob forward until the tablet breaks. Reading is noted down and is expressed in $\mathrm{kg} / \mathrm{cm}$.

\section{Thickness:}

The crown to crown thickness of tablets is measured by Vernier Caliper. It is expressed in $\mathrm{mm}$. the thickness variation allowed are $\pm 5 \%$ of the size of the tablet.

\section{Weight Variation:}

Twenty tablets were randomly selected from each batch and individually weighed. The average weight and standard deviation three batches were calculated. It passes the test for weight variation test if less than two of the individual tablet weights deviate from the everyday weight by quite than the allowed proportion deviation and none deviate by more than twice the proportion shown. It was calculated on an electronic weighing balance.

\section{Friability:}

The friability of the tablet can determined using Roche Friabilator. It is expressed percentage (\%). 10 tablets were at first weighed and transferred into the friabilator. The friabilator was operated at $25 \mathrm{rpm}$ for 4 minutes. The tablets were weighed again. And the $\%$ friability was calculated as.

\section{Friability $=([$ WO - W $] /$ WO $) 100$}

Where, WO = Initial weight of tablet.

$\mathrm{W}=$ after test weight of tablet.

\section{Disintegration Test:}

Six tablets were taken randomly from each batch and placed in USP disintegration apparatus baskets Apparatus was run for 10 minutes and the basket was lift from the fluid, observe whether all of the tablets have disintegrated.

\section{In-vitro Release:}

Drug release from liquisolid tablets was determined by using dissolution test apparatus United States Pharmacopoeia (USP) type II (paddle). $5 \mathrm{ml}$ aliquots of sample were withdrawn each time at suitable time intervals (5, 10, 15, 20, 25, 30, 45 and 60 minutes.) and replaced with fresh medium. After withdrawing, samples were filtered and analyzed after appropriate dilution by appropriate analytical method. The concentration was calculated using standard calibration curve.

\section{Uniformity of Drug content:}

The drug content can be determined by triturating sufficient amount of tablets and powder equivalent to average weight was added in $100 \mathrm{ml}$ of suitable buffer solution. Followed by stirring for $30 \mathrm{~min}$. Dilute suitably and the absorbance of resultant solution was measured spectrophotometrically ${ }^{18}$.

\section{Differential Scanning Calorimetry (DSC)}

Differential Scanning Calorimetry (DSC) is performed in order to assess the thermo tropic properties and the thermal behaviors of the drug, excipients used in the formulation of the liquisolid system. Complete disappearance of characteristic peaks of drug indicates the formulation of the drug solution in the liquisolid powder system, i.e., the drug is molecularly dispersed within the liquid matrix ${ }^{19}$.

\section{Fourier Transform Infrared Spectroscopy (FTIR)}

These studies are performed to estimate the chemical interactions between excipients and drug. If there's the presence of characteristic peaks and absence of additional peaks in formulation indicates that there are not any chemical interactions.

\section{Powder X-ray Diffraction (PXRD)}

The disappearance of characteristic peaks and holding of additional peaks of carriers within the liquisolid formulation is observed. It indicates that drug converted to amorphous form or to stabilized form ${ }^{20}$.

\section{APPLICATIONS OF LIQUISOLID COMPACTS ${ }^{21}$}

* Rapid release rates are obtained in liquisolid formulations.

* Liquisolid compact technology is a powerful tool to improve bioavailability of water insoluble drugs. Several water insoluble drugs on dissolving in different non-volatile solvents have been formulated into liquisolid compacts.

* These can be powerfully used for water insoluble solid drugs or liquid lipophilic drugs.

* Sustained release of drugs which are water soluble drugs such as propranolol hydrochloride has been obtained by the use of this technique.

* Solubility and dissolution enhancement.

* Flowability and compressibility.

* Designing of controlled release tablets.

* Application in probiotics. 


\section{ADVANTAGES OF LIQUISOLID SYSTEMS ${ }^{22}$}

* A countless number of slightly and very slightly watersoluble and practically water- insoluble liquid and solid drug can be formulated into liquisolid systems using the new formulation-mathematical mode

* Number of water-insoluble solid drug can be formulated into liquisolid systems.

* This technique is successfully applied for low dose water insoluble drug.

* Lower production cost than that of soft gelatin capsules.

* Drug dissolution from liquisolid compact is independent to the volume of dissolution media.

* Maximum of liquid or solid 'water-insoluble drug' may be formulated into immediate release or sustained release 'Liquisolid compact'

* Exhibits enhanced in-vitro and in-vivo drug release as compared to commercial counterparts, including soft gelatin capsule preparations.

* Can be used in controlled drug delivery.

* Increase bioavailability as compared to conventional tablets.

* Drug release can be modified using proper formulation ingredients.

\section{LIMITATIONS ${ }^{23}$}

$>$ Not applicable for the formulation of high dose insoluble drugs.

$>$ If more amount of carrier is added to produce free flowing powder, the tablet weight increases to more than one gram which is difficult to swallow.

$>$ Suitable compression properties might not be achieved since throughout compression liquid drug may be squeezed out of the liquid-solid tablet resulting in tablets of inadequate hardness.

$>$ Introduction of this method on industrial scale and to overcome the problems of mixing small quantities of viscous liquid solutions onto large amounts of carrier material may not be feasible.

\section{CONCLUSION}

In conclusion, the liquisolid system is that the new technique for the formulation of water insoluble drugs to improve their aqueous solubility, absorption still as dissolution rate, which leading to improvement of bioavailability of drugs as compared to conventional directly compressed tablets. Liquisolid formulations were designed to contain liquid medications in powdered form. The technique is also used hydrophilic carries in liquisolid systems. Therefore, increase the wetting properties and surface area of drug particles, and hence improve the dissolution profiles and might be oral bioavailability of the drug.

\section{REFERENCES:}

1. Yadav Y et al. A Review on Liquisolid Technique. Asian Journal of Chemical and Pharmaceutical Research.2014; 2(2):186-191.

2. Geethika et al. Liquisolid Compact Technology: A Review.Indo American Journal of Pharmaceutical Sciences. 2015; 2(3):684-691.

3. Patel N. Liquisolid Dosage System: A Novel Approach for Dosage formulation. Journal of Pharmaceutical Science and Bioscintific Research.2014; 4(1):125-130.
4. Spiras S. Liquisolid systems and methods for preparing same, United States patent 6, 423,339 B1, 2002.

5. Spiras S, Bolton S.M. Liquisolid systems and methods for preparing same, United States patent 6,096,337, 2000.

6. Vajir S, Sahu V, Ghuge N and Bakde B.V. Effect of Dissolution Rate by Liquisolid Compact Approach: An Overview. International Journal of Pharmaceutical and Chemical Sciences.2012; 1(3):1013-1018.

7. Nagabandi VK, Ramarao T, Jayaveera K.N. Liquisolid Compacts: A Novel Approach to Enhance Bioavailability of Poorly Soluble Drugs. International Journal of Pharmacy and Biological Sciences.2011; 1(3):89-102.

8. Kala N.P., Shaikh M.T., Shastri D. H., Shelat P.K.A., Review on Liquisolid Systems. Journal of Drug Delivery and Therapeutics; 2014; 4(3):25-31.

9. Sirisha V.N.L. et al. A Review on Liquid Solid Compacts. International Journal of Pharmaceutical and Phytopharmacological Research. 2012; 2(2): 116-121.

10. Dalvi P.B and Ingale P.R. Liquisolid Technique: An Approach for Enhancement of Solubility. World Journal of Pharmacy and Pharmaceutical Sciences.2014; 3(12):434-446.

11. Jadhav E.L and Geeverghese R. Liquisolid Technique for Solubility Enhancement: Review Article. World Journal of Pharmaceutical Research.2015; 4(4):1756-1767.

12. Patel K.J, Patel Y.K. Liquisolid Technique: Enhancement of Solubility and Dissolution Rate: A Modern Review. International Journal of Pharmaceutical Research and BioScience. 2014; 3(2):397-407.

13. Jadhav et al. Liquisolid Compact: A New Technique for Enhancement of Drug Dissolution. International Journal of Research in Pharmacy and Chemistry.2011; 1(3):705-713.

14. Aneena G.M, Krishnakumar K, Kavitha M.P. Liquisolid System: A Review. International Journal of Pharmaceutical Development \& Technology.2016; 6(2):80-83.

15. Fahmy R.H, Kassem M.A. Enhancement of Famotidine dissolution rate through liquisolid tablet formulation: In vitro and In vivo evaluation. Eur. J. Pharm. Biopharm. 2008; 69:993- 1003.

16. Kharwade M, and Sneha M. A Review on Pioneering Technique - Liquisolid Compact and Applications. Research Journal of Pharmaceutical, Biological and Chemical Sciences.2015; 6(2):220-227.

17. Rajesh K, Rajalakshmi R, Umamaheswari J, C.K. Ashok Kumar. Liquisolid Technique A Novel Approach To Enhance Solubility And Bioavailability. International Journal of Biopharmaceutics. 2011; 2(1): 8-13.

18. Chandel P, Kumari R, Kapoor A. Liquisolid Technique: An Approach for Enhancement of Solubility. Journal of Drug Delivery and Therapeutics.2013; 3(4):131-137.

19. Pradeep Kumar C.H, Venugopalaiah P, Praveen Kumar, Gnanaprakash K, Gobinath M. Liquisolid Systems - An Emerging Strategy For Solubilization \& Dissolution Rate Enhancement of BCS Class-II Drugs. International Journal of Pharmacy Review \& Research.2013; 3(2):56-66.

20. Balaji A, Umashankar M.S and Kavitha B. Liquisolid Technology- A Latest Review. International Journal of Applied Pharmaceutics.2014; 6(1):11-19.

21. Nagabandi V.K, Ramarao T, Jayaveera K.N. Liquisolid Compacts: A Novel Approach to Enhance Bioavailability of Poorly Soluble Drugs. International Journal of Pharmacy and Biological Sciences.2011; 1(3):89-102.

22. Sandip, et al. Liquisolid Compact: Liquisolid Compact: A Novel Approach To Enhance Bioavailability of Poorly Soluble Drug. International Journal of Pharmacy.2012; 2(3):586-590.

23. Cherukuri Sowmya et al. Liquisolid Compact: A Novel Approach to Enhance Bioavailability of Poorly Soluble Drug. International Research Journal of Pharmacy.2012; 3(7):108115 . 